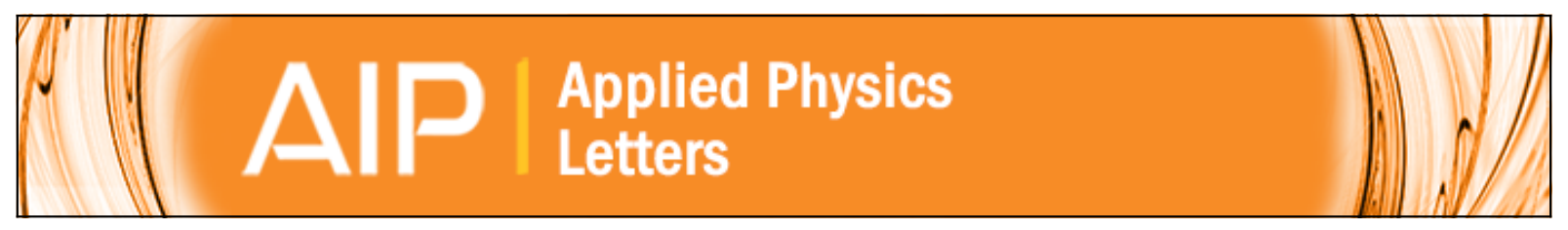

\title{
Geomagnetic sensor based on giant magnetoelectric effect
}

Junyi Zhai, Shuxiang Dong, Zengping Xing, Jiefang Li, and D. Viehland

Citation: Applied Physics Letters 91, 123513 (2007); doi: 10.1063/1.2789391

View online: http://dx.doi.org/10.1063/1.2789391

View Table of Contents: http://scitation.aip.org/content/aip/journal/apl/91/12?ver=pdfcov

Published by the AIP Publishing

\section{Over 700 papers \&} presentations on multiphysics simulation vew Now

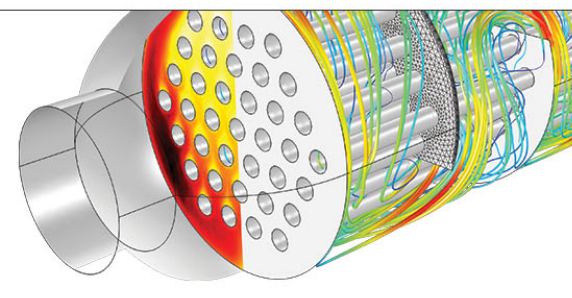




\title{
Geomagnetic sensor based on giant magnetoelectric effect
}

\author{
Junyi Zhai, ${ }^{\text {a) }}$ Shuxiang Dong, Zengping Xing, Jiefang Li, and D. Viehland \\ Department of Materials Science and Engineering, Virginia Tech, Blacksburg, Virginia 24061, USA
}

(Received 25 June 2007; accepted 2 September 2007; published online 20 September 2007)

\begin{abstract}
Here, the authors report a new type of geomagnetic field sensor based on the giant magnetoelectric effect in Metglas/piezoelectric-fiber laminates that are wrapped with a coil. These sensors can measure quite precisely the value of both the Earth's magnetic field and its inclination. The geomagnetic field sensor does not require a dc magnetic bias and is driven by a $10 \mathrm{~mA}$ ac. Highly sensitive dc magnetic field variations of less than $10^{-9} \mathrm{~T}$ and angular inclinations of $\leqslant 10^{-5} \mathrm{deg}$ can be detected, potentially offering opportunities for a small global positioning device.
\end{abstract}

(C) 2007 American Institute of Physics. [DOI: 10.1063/1.2789391]

Migratory animals are capable of sensing variations in geomagnetic fields as a source of guidance information during long-distance migration. ${ }^{1,2}$ It is well known that geomagnetic fields are on the order of 0.4-0.6 Oe and have different inclinations at different locations. The Earth's mean field and its inclinations at many points over much of the Earth's surface, including spatial variations and temporal evolutions, have been tabulated by the United States Geological Survey. ${ }^{3}$ Accordingly, geomagnetic field sensors could be used in guidance and positional location.

There are many types of magnetic sensors: for example, superconducting quantum interference devices ${ }^{4}$ or giant magnetoresistance spin valves. ${ }^{5}$ However, these sensors require very low operational temperatures (liquid nitrogen) in order to achieve high sensitivity. Fluxgate sensors ${ }^{6-8}$ based on exciting coil/high permeability core/pick-up coils have been investigated for many years to detect dc magnetic and geomagnetic fields. This widely used sensor is relatively cheap and temperature independent; however, its magnetic hysteresis, offset value under zero magnetic field, and large demagnetization factor restrict design considerations.

Recently, new types of passive ac (Ref. 9) and active dc (Ref. 10) magnetic field sensors have been developed based on a giant magnetoelectric (ME) effect-which are simple devices that work at room temperature. The ME property tensor changes a magnetic induction into an induced polarization. Although the $\mathrm{ME}$ effect was first reported in $\mathrm{Cr}_{2} \mathrm{O}_{3}$ crystals in the $1950 \mathrm{~s},{ }^{11}$ the values of the ME property coefficient have always been found to be extremely small for single phase materials (i.e., $\leqslant \mathrm{mV} / \mathrm{cm} \mathrm{Oe}$ ). However, since 2001, laminated composites, such as magnetostrictive Terfenol-D or ferrite layers epoxied together with $\mathrm{Pb}\left(\mathrm{Zr}_{1-x}, \mathrm{Ti}_{x}\right) \mathrm{O}_{3}(\mathrm{PZT})$ ones, ${ }^{12-17}$ have been found to possess giant $\mathrm{ME}$ effects of between 0.1 and $2 \mathrm{~V} / \mathrm{cm}$ Oe under dc magnetic bias of $H_{\mathrm{dc}}<500 \mathrm{Oe}$. Furthermore, "even larger giant" ME coefficients of up to $22 \mathrm{~V} / \mathrm{cm}$ Oe under $H_{\mathrm{dc}}$ $<5$ Oe have recently been reported for Metglas/PZT-fiber laminates at quasistatic frequency, ${ }^{18}$ which is $>10$ times larger than prior reports for laminates ${ }^{12-17}$ and $>10^{4}$ times larger than that of single phases. ${ }^{11}$ In this letter, we will show by using a Metglass/PZT-fiber ME sensor that we can precisely detect both geomagnetic fields and their inclinations along various axes of a globe.

${ }^{a)}$ Electronic mail: zhjy@vt.edu
Our ME sensor is a Metglas/PZT-fiber laminate with a 100 circle coil wrapped tightly around it, as illustrated in Fig. 1(a). This laminate configuration is similar to that which we recently reported. ${ }^{18}$ The PZT fibers were $200 \mu \mathrm{m}$ in thickness and were laminated between four layers of Metglas by use of a thin layer epoxy; the thickness of each Metglas layer was $25 \mu \mathrm{m}$, and the total dimensions of the laminates were $100 \times 6 \times 0.48 \mathrm{~mm}^{3}$. The working principal of the ME sensor is that an input magnetic field changes the length of the Metglas via magnetostriction, and because the PZT fibers are elastically bound to the Metglas layers through an epoxy interfacial layer, the PZT fibers also change their length and generate an output voltage via piezoelectricity. Detection of the Earth's magnetic field was performed by applying a $1 \mathrm{kHz}$ ac magnetic field $\left(H_{\mathrm{ac}}\right)$ via a $10 \mathrm{~mA}$ ac input to the coil and by measuring the dc voltage and its phase induced in the PZT fibers by a lock-in amplifier (SR-850).

Figure 1 shows the dependence of the induced ME voltage $\left(V_{\mathrm{ME}}\right)$ on $H_{\mathrm{dc}}$. Over the range of $-1.5<H_{\mathrm{dc}}<1.5 \mathrm{Oe}$, $V_{\mathrm{ME}}$ was linearly proportional to $H_{\mathrm{dc}}$ and equal to $300 \mathrm{mV}$ under a $H_{\mathrm{dc}}=1 \mathrm{Oe}$ - which was $>10^{3}$ times that of a corresponding Terfenol-D/PZT dc magnetic field sensor operated at $1 \mathrm{kHz} .{ }^{10}$ Another important finding was that, unlike Terfenol-D/PZT magnetic sensors, ${ }^{10} V_{\mathrm{ME}}$ for Metglas/PZTfiber sensors was not dependent on $H_{\mathrm{dc}}$ history (i.e., no hysteretic phenomena): rather, $V_{\mathrm{ME}}$ only depended on momen-

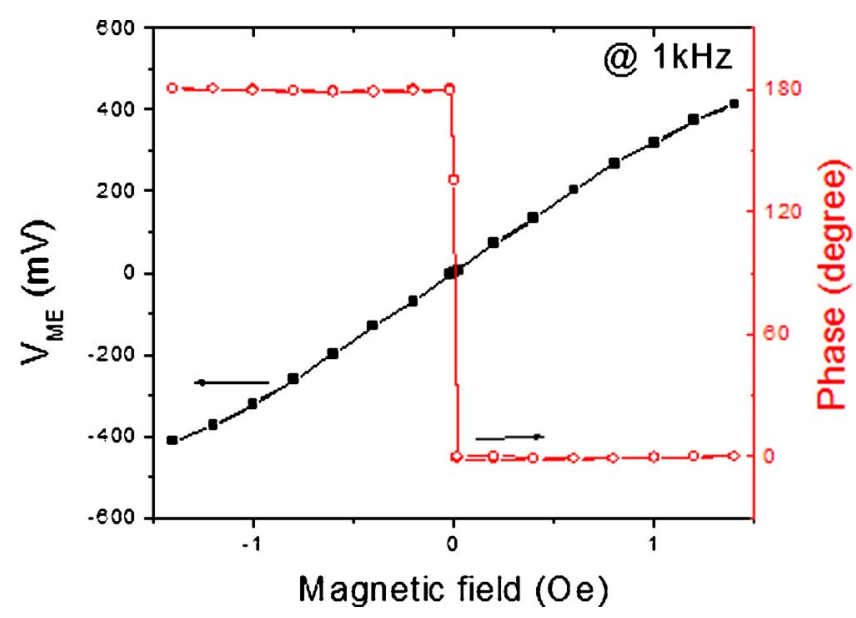

FIG. 1. (Color online) dc magnetic field dependence of the ME voltage coefficient and phase for a Metglas/PZT-fiber push-pull laminate, measured at $1 \mathrm{kHz}$ under an applied current of $10 \mathrm{~mA}$. 




FIG. 2. (Color online) Photograph of the geomagnetic field sensor measurement setup.

tary changes of $H_{\mathrm{dc}}$ in the environment. This is very important to a stable and repeatable detection of dc magnetic fields and their variations. In addition, when the sign of $H_{\mathrm{dc}}$ was changed, a dramatic $180^{\circ}$ phase shift was found. This shift could be used to distinguish the direction along which changes in $H_{\mathrm{dc}}$ occur with respect to the length (long axis) of the sensor. This is an important advantage compared to fluxgate. Previously, ${ }^{18}$ it was reported that $V_{\mathrm{ME}}$ from a Metglas/ PZT fiber laminates was strongly anisotropic, offering good sensitivity to magnetic field variations only along its length direction. In the other two perpendicular directions, only very weak signals were found with changes in $H_{\mathrm{dc}}$. These unique properties of Metglas/PZT-fiber ME sensors are due to the ultrahigh relative permeability $\left(\mu_{r}\right)$ of Metglas, which is $>10^{3}$ times larger than that of Terfenol-D or nickel ferrite. Correspondingly, the high $\mu_{r}$ of Metglas results in an ultrasmall demagnetization field, enabling a high effective piezomagnetic coefficient at low biases.

Figure 2 illustrates our experimental setup for measuring the Earth's magnetic field. The globe was mounted on a three-axis circle, enabling rotation in all directions. Our ME sensor was placed inside of the globe on an aluminum holder. A lock-in amplifier was used to generate a $1 \mathrm{kHz}$ ac, which was used as an input to the coil wrapped around the sensor and which was also used as a reference signal for detection. During measurements, we first rotated the globe about the up-down axis inside of the Earth's plane; subsequently, we rotated about the north-south axis and then about the east-west one. Each time we rotated by an angle of $5^{\circ}$. Please note that our designation of the north and south directions is not that of the Earth's direction but rather that of the magnetic north and south ones.

Figure 3(a) shows the angular dependence of $V_{\mathrm{ME}}$ when the sensor is rotated in the Earth's plane. As expected, when the sensor was oriented along the east and west directions in this plane, its output was essentially $0 \mathrm{mV}$; whereas when it was oriented along the south and north directions in the plane, its output was maximum. This is because our ME sensor is only sensitive along its length direction; thus, when this direction is oriented along the geomagnetic field in Earth's plane, it has only vector components in the northsouth axis. Figure 3(b) shows $V_{\mathrm{ME}}$ when the sensor is rotated along the north-east axis. Similar to Fig. 3(a), when the sen-


FIG. 3. (Color online) Output voltage from the magnetoelectric sensor when it is rotated (a) in the Earth's plane, (b) along the north-south axis, and (c) along the east-west axis.

sor was oriented along the east-west direction, $V_{\mathrm{ME}}$ was nearly $0 \mathrm{mV}$; whereas the other two directions, up and down, had the largest sensor output. Figure 3(c) shows $V_{\text {ME }}$ when the sensor is rotated along the east-west axis. The inclination 
of our laboratory (Blacksburg, VA) in this plane was $65^{\circ} 51^{\prime}$. Our experimental results showed that when our sensor angle was set at $65^{\circ}$ and/or $235^{\circ}$, the output voltage was also the largest in this plane.

The sensitivity of our Metglas/PZT-fiber laminates to changes in dc magnetic field was $H_{\mathrm{dc}}=0.8 \mathrm{nT}$ over a range of operations of $\pm 1.5 \mathrm{Oe}$. These properties are comparable to the fluxgate sensor. ${ }^{8}$ Along a set plane, changes in the angle of rotation will result in changes in the geomagnetic field vector. The best angular sensitivity was found along the eastwest direction, which was $\approx 3 \times 10^{-6} \mathrm{deg}$; whereas, the worst sensitivity was found along the north-south direction, which was $\approx 10^{-5} \mathrm{deg}$. Based on these results, one could develop a three-axis ME sensor unit, where each sensor is perpendicular to the other two. Using the said three-axis ME sensor unit, one could determine the local magnetic field and its inclination by knowing the geomagnetic field. Accordingly, a small global positioning device could be made with quite good accuracies by comparing to a grid of values for Earth's field and its spatial/temporal variations.

In summary, we have developed a geomagnetic field sensor based on the giant magnetoelectric effect in Metglas/ PZT-fiber laminates; this sensor does not need dc bias to achieve an optimum performance but rather is driven by a small ac. Our sensor has good anisotropy in the magnetic field direction, and a high sensitivity to variations in Earth's field of $H_{\mathrm{dc}}=0.8 \mathrm{nT}$. These low cost and low power consuming laminates (with wrapped coil) offer potential applications in global positioning.
We gratefully acknowledge full supported by the Office of Naval Research.

${ }^{1}$ W. Cochran, H. Mouritsen, and M. Wikelski, Science 304, 405 (2004).

${ }^{2}$ K. J. Lohmann, C. M. F. Lohmann, L. M. Ehrhart, D. A. Bagley, and T. Swing, Nature (London) 428, 909 (2004).

${ }^{3}$ Please see http://www.ngdc.noaa.gov/seg/geomag/jsp/struts/ calcIGRFWMM

${ }^{4}$ J. Vrba, SQUID Sensors: Fundamentals, Fabrication and Applications, edited by H. Weinstock (Kluwer, Dordrecht, 1996), p. 117.

${ }^{5}$ M. N. Baibich, J. M. Broto, A. Fert, F. N. Van Dau, F. Petroff, P. Eitenne, G. Creuzet, A. Friederich, and J. Chazelas, Phys. Rev. Lett. 21, 2472 (1988).

${ }^{6}$ D. I. Gordon, IEEE Trans. Magn. Mag-8, 76 (1972).

${ }^{7}$ J. E. Lenz, Proc. IEEE 78, 973 (1990).

${ }^{8}$ P. Ripal, Sens. Actuators, A 33, 129 (1992).

${ }^{9}$ J. Y. Zhai, S. X. Dong, J.-F. Li, and D. Viehland, Appl. Phys. Lett. 88, 062510 (2006).

${ }^{10}$ S. X. Dong, J. Y. Zhai, J.-F. Li, and D. Viehland, Appl. Phys. Lett. 88, 082907 (2006).

${ }^{11}$ I. E. Dzyaloshinskii, Sov. Phys. JETP 10, 628 (1959).

${ }^{12}$ S. X. Dong, J.-F. Li, and D. Viehland, Appl. Phys. Lett. 83, 112265 (2003).

${ }^{13}$ M. I. Bichurin, R. V. Petrov, and Y. V. Kiliba, Ferroelectrics 204, 311 (1997).

${ }^{14}$ G. Srinivasan, E. T. Rasmussen, J. Gallegos, R. Srinivasan, Yu. I. Bokhan, and V. M. Laletin, Phys. Rev. B 64, 214408 (2001).

${ }^{15}$ C.-W. Nan, L. Liu, N. Cai, J. Zhai, Y. Ye, Y. H. Lin, L. J. Dong, and C. X. Xiong, Appl. Phys. Lett. 81, 3831 (2002).

${ }^{16}$ J. Y. Zhai, N. Cai, Z. Shi, Y. H. Lin, and C.-W. Nan, J. Appl. Phys. 95, 5685 (2004).

${ }^{17}$ G. Srinivasan, E. T. Rasmussen, B. Levin, and R. Hayes, Phys. Rev. B 65 , 134402 (2002)

${ }^{18}$ S. X. Dong, J. Y. Zhai, J.-F. Li, and D. Viehland, Appl. Phys. Lett. 89, 252904 (2006). 